TEI

JOURNAL OF THE
Journal of the Text Encoding Initiative

Issue 8 | December 2014 - December 2015

Selected Papers from the 2013 TEI Conference

\title{
Editorial Introduction to Issue 8 of the Journal of the Text Encoding Initiative
}

Arianna Ciula and Fabio Ciotti

\section{OpenEdition}

\section{Journals}

Electronic version

URL: http://journals.openedition.org/jtei/1380

DOI: 10.4000/jtei.1380

ISSN: 2162-5603

Publisher

TEl Consortium

\section{Electronic reference}

Arianna Ciula and Fabio Ciotti, «Editorial Introduction to Issue 8 of the Journal of the Text Encoding Initiative », Journal of the Text Encoding Initiative [Online], Issue 8 | December 2014 - December 2015, Online since 07 December 2015, connection on 22 September 2020. URL : http:// journals.openedition.org/jtei/1380 ; DOI : https://doi.org/10.4000/jtei.1380

For this publication a Creative Commons Attribution 4.0 International license has been granted by the author(s) who retain full copyright. 


\title{
Editorial Introduction to Issue 8 of the Journal of the Text Encoding Initiative
}

\author{
Arianna Ciula and Fabio Ciotti
}

1 We are pleased to introduce the eighth issue of the Journal of the Text Encoding Initiative featuring selected peer reviewed papers from the 2013 TEI Conference and Members Meeting, which was held at Università della Sapienza in Rome, 2-5 October.

2 Like all TEI conferences, this was an international gathering and, compared to other conferences, enjoyed an exceptional and diverse range of participants. The collaboration between the TEI Board of Directors and the Associazione Italiana di Informatica Umanistica e Cultura Digitale (AIUCD) to ensure an Italian host for this event also aimed at marking the long standing involvement of the Italian Digital Humanities community in TEI. Indeed, Italian scholars have made considerable contributions to the development of TEI and Digital Humanities as a whole in terms of theory, technologies, and organisation. We must also mention the name of the late Antonio Zampolli, who contributed enormously to the conception and implementation of the TEI initiative and who hosted the first Members' Meeting of the Consortium in Pisa in 2001. Since the 1990s scholars at Università della Sapienza in Rome have played an equally important role in raising the awareness 
of the importance of TEI encoding for formal textual representation. In particular, thanks to the research group led by the late Giuseppe Gigliozzi, research and teaching activities related to TEI have spread throughout Italy.

3 The theme of the conference-“The Linked TEI: Text Encoding in the Web"-focused on the role of text encoding and TEI in a networked cultural environment. This focus encouraged reflections on the semantics of the TEI conceptual model, on the relationships among TEI and other data models and encoding schemas, on the role of TEI within a framework of interconnected digital resources as well as on the diverse ways users can access and take advantage of TEI-encoded resources. The title hints obviously at a very topical theme in the digital realm: the emergence and diffusion of the Linked Data paradigm and of a Participatory Web. TEI has had a crucial-and widely recognised -role in encouraging and facilitating the creation of vast quantities of textual and linguistic resources. It has been one of the major enabling technologies in the Digital Humanities. However, the dominant paradigm in the creation of digital resources, especially in the academic domain, has been that of the individual archive, the single monolithic or boutique project, perfectum in itself. To continue in its role of stimulus for innovation in the Digital Humanities, TEI has to be able to embrace fully-albeit critically-the new paradigm. The sharing and the interconnection of data on the Web as well as the emergence of semantically-enriched data are interesting aspects of technological innovation which will bring about new developments. The vision around "Linked TEI" also encompasses issues of multilingualism and multiculturalism: to be connected means to recognise and collaborate with different traditions and languages.

4 Contributions to the conference call for papers responded very well to the challenge with a rich range of topics and perspectives represented in the programme: from reflections on semantic models and textuality, to data modelling and analysis, from re-thinking research infrastructures and developing participatory approaches, to establishing bi-directional linking between dictionaries and corpora. Among the many papers, posters, and panels accepted and presented at the conference, a large number were submitted for consideration and peer review for this issue of the Journal of the Text Encoding Initiative, resulting in the largest issue of the journal published to date.

$5 \quad$ The set of articles published here follow three main threads of interests around the TEI:

1. TEI in relation to other semantic and modeling formalisms; 
2. TEI as an expression of domain-specific text and data models;

3. TEI processing workflows and tools.

6 The first topic is opened by Ciotti, Lana, and Tomasi's article. In line with the main theme of the conference, this paper describes the architecture of the geolat project, a digital library of Latin texts enriched with annotations related to places and persons. Based on different ontologies and Semantic Web languages, these annotations can be formally analysed and shared as Linked Open Data. Also within the context of linked environments, Berti et al. introduce the Leipzig Open Fragmentary Texts Series project, the goals of which are the creation of linked and annotated digital editions of classical fragmentary texts and the development of the Perseids Platform, a collaborative environment for crowdsourced annotations. Moving from practical use-cases to the theoretical consideration of diverse modeling frameworks, Eide's and Piez's papers reveal the constraints of the tools we use as well as the different views they cast on the material under study. In particular, Piez presents the non-hierarchical modelling language LMNL (the Layered Markup and Annotation Language) and how it can be combined with the TEI-XML formalism to enhance its representational capability. Eide describes different methods of establishing meaning in TEI documents via linking mechanisms between TEI and external ontologies and how these different methods determine the semantic openness and usability of TEI documents.

7 The second cluster collects a set of articles related to the adaptation of the TEI encoding scheme to specific complex data and document types. Using the Shelley-Godwin Archive (S-GA) as a case study, Muñoz and Viglianti discuss the challenges of producing TEI-encoded data and an accompanying reading environment able to support both document-focused and text-focused approaches. Barbero and Trasselli describe the data model developed by the Instituto Centrale per il catalogo Unico (ICCU) to export manuscript descriptions from Manus OnLine (MOL), the Italian national catalogue of manuscripts, to TEI documents to promote consistency in manuscript descriptions as well as data interchange. Haaf, Geyken, and Wiegand give an overview of the DTA “Base Format" (DTABf), a strict subset of the TEI P5 tag set developed by the Deutsches Textarchiv (DTA) project to represent text types in historical corpora generated out of multiple printed sources. González-Blanco and Rodríguez discuss the use of the TEI Verse module to tag metrical and poetic structures in the Repertorio Digital de Métrica Medieval Castellana (ReMetCa) 
project, where TEI-XML is integrated into a Relational Database Management System (RDBMS). Last but not least, Mörth et al. propose a customization of the TEI dictionary module to respond to the new requirements emerging from an environment of increasingly intertwined language resources, and in particular to the need to include statistical data obtained from language resources in lexicographic workflows.

8 The contributions within the third set of papers describe various approaches and tools to create, manage, and disseminate TEI-encoded resources in a networked environment. De la Iglesia and Göbel's work uses XML and related Web technologies to process semantic entities and produce data-driven visualizations of the forthcoming edition of Theodor Fontane's notebooks as well as comparisons with other edition projects using TEI. Rosselli Del Turco et al. describe EVT (Edition Visualization Technology), a TEI based scholarly publishing tool under development since about 2010. Initially designed as a specific solution for the Digital Vercelli Book project, EVT has evolved into a flexible tool to create Web-based digital editions incorporating transcription files encoded in TEI XML and digital facsimiles of the source texts. Portela and Rito Silva's article presents the rationale and the technical approaches adopted for the digital edition of Fernando Pessoa's Livro do Desassossego (LdoD). Starting from the intrinsic complexity of this unfinished work, this project offers a digital model of all its authorial and editorial manifestations and aims to create a dynamic platform open to user interaction and collaboration. Dumont and Fechner's paper illustrates the user-friendly and bottom-up design principles that have guided the development of the ediarum TEI editing and publishing platform. Similarly, Boschetti and Del Grosso describe the design and development of TeiCoPhiLib, a library of Java software components devoted to editing, processing, and visualising TEI documents in the domain of philological studies. This tool is particularly suited to fostering collaborative philological work. This cluster is closed by Dalmau and Hawkins's article, the focus of which is not on specific tools or platforms, but rather on the impact and adoption of TEI and text encoding practices in the library community.

9 We conclude this introduction with a few words about how this issue fits within the current developments of TEI and of the Digital Humanities community more generally. Every now and then it is healthy to ask where the TEI is going and why we care about it. With the growing emergence of Digital Humanities curricula and research positions, the establishment of digital workflows and resources in the cultural heritage sector, and the ongoing transition from a print to digital scholarly 
communication cycle, it is easy to get caught in the vortex. With the flurry of new activity in Digital Humanities today, has the time and place for the TEI come and gone? A step back to think of where we are-and therefore where we are going-implies a reconsideration of the historical focus of the TEI: texts, and in particular the modeling of texts for representation and processing purposes. It is this historical focus together with a periodical shifting of its limits and limitations that ensures the continuing relevance of the TEI: the meanings of texts change; the term "text" itself is perceived and applied in a wide sense; what we want to do with texts changes. The heart of our research endeavours continues to be the slippery confines of our cultural productions, to be seen, analysed, reflected upon, deconstructed, formalized, processed, remediated, and re-interpreted.

10 The key to ensuring that the TEI will continue its valuable contributions to scholarship and culture more generally lies therefore also in a slight but crucial tilt to its rhetorics: the TEI is not only about delivering a standard but rather, and perhaps more significantly, about the intellectual activity of creating and developing that standard in partnership with the diverse communities of researchers, archivists, librarians, and other professionals of the cultural heritage sector, software developers, infrastructures providers, artists, citizens. A linked TEI looks less and less like a jigsaw where all pieces are cut to fit together, and more like an intertwining of hands. Enjoy the reading.

\section{AUTHORS}

\section{ARIANNA CIULA}

Arianna Ciula is Research Facilitator at University of Roehampton (London, UK), where she supports research and enterprise strategies and actively contributes to the research profile and networks in particular of the Department of Humanities. She is the secretary of EADH, member of the ADHO Steering Committee and member of CLARIN Scientific Advisory Board. She served in the TEI Technical Council as well as TEI Board. 


\section{FABIO CIOTTI}

Fabio Ciotti is Assistant Professor at the University of Roma Tor Vergata, where he teaches Digital Literary Studies and Theory of Literature. He is President of the Associazione per l'Informatica Umanistica e la Cultura Digitale (AIUCD, the Italian digital humanities association) and member of the EADH Executive Board. He has been scientific consultant for text encoding and technological infrastructures in several digital libraries and archives projects, and has served in the TEI Technical Council. 